\title{
AMPLITUDE MODULATION OF RADIAL P-MODES FROM VIRGO
}

\author{
T. LEIFSEN \\ Institute of Theoretical Astrophysics, PO Box 1029, Blindern, \\ N-0315 Oslo, Norway \\ B.N. ANDERSEN \\ Norwegian Space Centre, PO Box 113 Skøyen, N-0212 Oslo, Norway
}

AND

THE VIRGO TEAM

\begin{abstract}
The radial order solar $p$-modes show an amplitude modulation at the equatorial siderial rotation frequency of the Sun. The peak is clearly separated from the peak at the synodic rotation visible in the irradiance data.
\end{abstract}

\section{Data and analysis}

In this study a continuous dataset of solar irradiance sampled every 60 seconds with the VIRGO SPM blue channel on the SOHO spacecraft for the time period 29-01-96 to 23-07-97 has been used to study the time and frequency variation of the solar $l=0 p$-modes. Also the Stanford index of solar global magnetic field from 29-01-96 to 30-06-97 was used. Wavelet analysis with a modified Morlét wavelet as basis function was used to study the time variation of the amplitudes of the $l=0 p$-modes with radial orders of 12-32. The different modes have been studied using a variety of combination of temporal and frequency resolution. The amplitude modulation was studied using temporal resolution of about half a day. The $l=0$ data have been filtered in the frequency domain to remove the effects of the nearby $l=2$ modes. Supercomputing time was provided by The Research Council of Norway.

\section{Results and Conclusions}

The power spectra of the SPM data and magnetic index values show a clear peak at the solar synodic rotation frequency. The power spectrum of the radial order averaged $(n=14-26) l=0$ mode amplitudes show a clear peak at the solar siderial rotation frequency. Correcting for the siderial/synodic effect in the sampling of the mode amplitudes makes the rotation peak coincident with the peaks of the SPM data and the magnetic index. In addition the power around $1 \mu \mathrm{Hz}$ is reduced due to removal of the phasedrift caused by the orbital motion of the Earth. The autocorrelation function of the SPM data has twice the width of the magnetic index and the mode amplitudes. This is due to the opposite center to limb variation of sunspots and faculae. The 


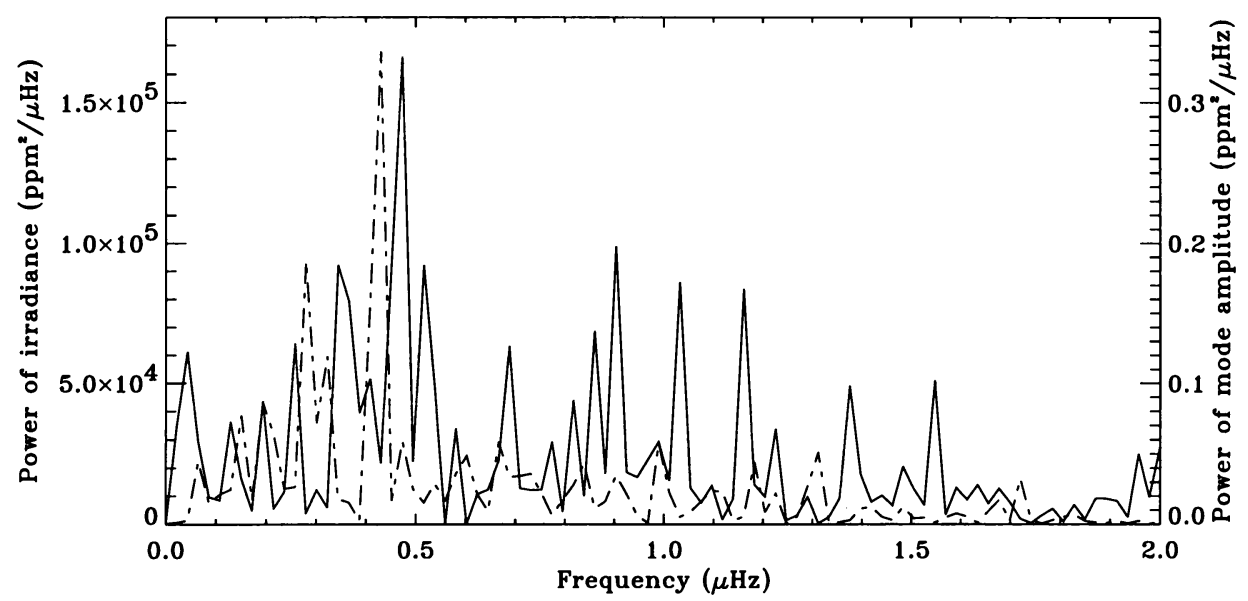

Figure 1. The power spectrum of the amplitude (averaged over $n=16-26$ ) of $l=0$ (solid line) and the power spectrum of the intensity in the SPM blue channel (dashed line).
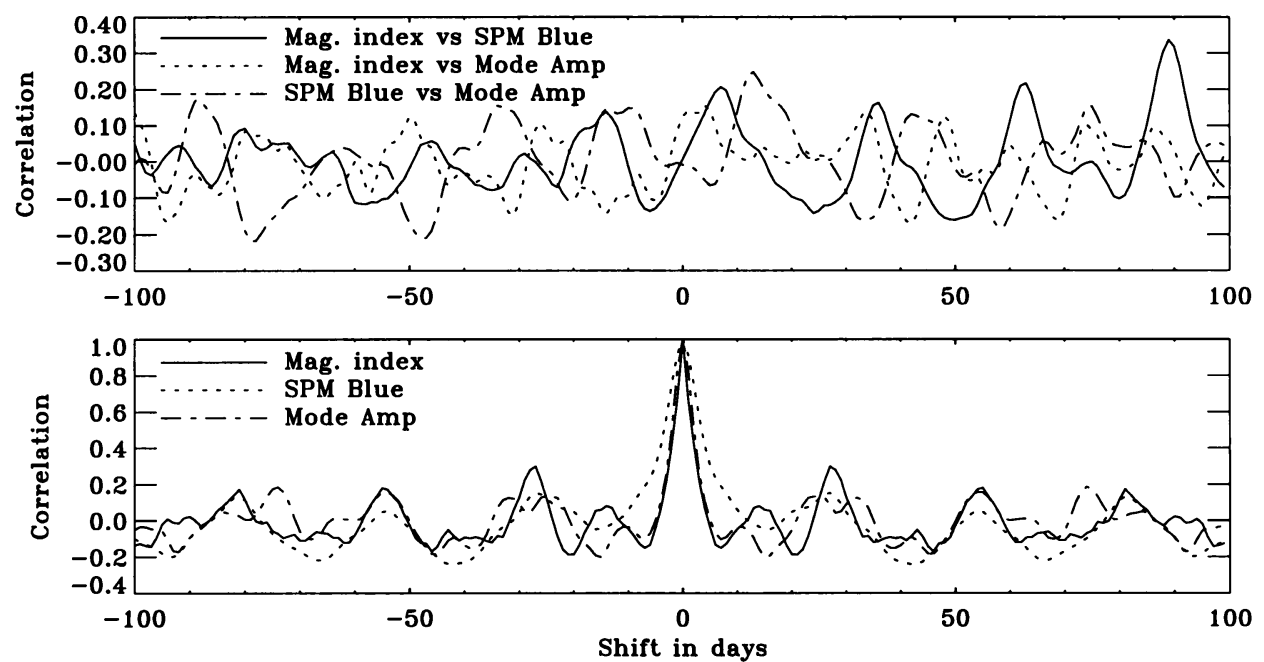

Figure 2. The panels show the cross- (upper) and autocorrelations of the timestrings as given in the legends. Mode amplitude timestrings are resampled synodically.

synodically sampled mode amplitudes averaged for $n=14-26$ are nearly in phase with the magnetic index, while there is a weak anticorrelation with the SPM irradiance. The temporal and frequency behaviour of the $l=0$ modes are very structured with accumulated pulses down to the time resolution of half a day.

The mode amplitudes of the $l=0 p$-modes are strongly modulated by the siderial rotation of the Sun. This modulation is not associated with the meridian passing of the active regions/magnetic fields. This is clearly seen in the direct power spectra where the peaks are separated by a frequency difference corresponding to the siderial/synodic effect. However, the amplitude of the rotational modulation is clearly dependent on the amount of solar activity on the visible hemisphere. 